\title{
Aerobic Exercise and Metabolic Syndrome: The Role of Sympathetic Activity and the Redox System
}

\author{
Vincenzo Monda' \\ Francesco Sessa $\mathbb{1 D}^{2}$ \\ Maria Ruberto ${ }^{3}$ \\ Marco Carotenuto (D) ${ }^{4}$ \\ Gabriella Marsala (D) $^{5}$ \\ Marcellino Monda' \\ Maria Teresa Cambria ${ }^{6}$ \\ Marinella Astuto ${ }^{7}{ }^{7}$ \\ Alfio Distefano ${ }^{6}$ \\ Giovanni Messina ${ }^{2}$ \\ 'Department of Experimental Medicine, \\ Università degli Studi della Campania \\ "Luigi Vanvitelli", Caserta 8I I00, Italy; \\ ${ }^{2}$ Department of Clinical and \\ Experimental Medicine, University of \\ Foggia, Foggia 7II2I, Italy; ${ }^{3}$ CDR Santa \\ Maria del Pozzo, Naples 80049, Italy; \\ ${ }^{4}$ Clinic of Child and Adolescent \\ Neuropsychiatry, Department of Mental \\ Health, Physical and Preventive Medicine, \\ Università degli Studi della Campania \\ "Luigi Vanvitelli", Caserta 8I 100, Italy; \\ ${ }^{5}$ Struttura Complessa di Farmacia, \\ Azienda Ospedaliero-Universitaria, \\ Ospedali Riuniti di Foggia, Foggia 71/21, \\ Italy; 'Section of Medical Biochemistry, \\ Department of Biomedical and \\ Biotechnological Sciences, University of \\ Catania, Catania 95I23, Italy; ${ }^{7}$ Azienda \\ Ospedaliera "Policlinico Vittorio \\ Emanuele", U.O. di Anestesia e Terapia \\ Intensiva, Catania $95 / 23$, Italy
}

This article was published in the following Dove Press journal: Diabetes, Metabolic Syndrome and Obesity: Targets and Therapy
Background: Aerobic exercise can greatly assist in reducing collateral effects of metabolic syndrome (MetS). Moreover, aerobic exercise is associated with sympathetic activation and adaptive responses to sustain muscle engagement, changes in the release of Orexin A, a pleiotropic neuropeptide.

Aim: The aim of this study was to analyze the beneficial effects of aerobic exercise without dietary changes, in a cohort of MetS subjects, focusing on the role of sympathetic and orexinergic activity. Several blood parameters linked to MetS ROS production, heart rate, galvanic skin response, d-ROM test, and Orexin A serum levels were evaluated in ten males with MetS (BMI 30-34.9) before and after a period of 6 months of aerobic exercise compared to ten healthy subjects.

Methods: Ten male subjects (aged $54 \pm 4.16$ ) with MetS (MetS group) and ten healthy males (aged $49.7 \pm 2.79$, Healthy group) were told about the study protocol and possible risks, signed the informed consent, and voluntarily participated in the study. Several blood parameters were evaluated in the two tested groups and were re-evaluated in the MetS group after 6 months of training (MetS6M group). The training protocol consisted of more than 30 $\mathrm{min} /$ day of walking (average speed of $4.5 \mathrm{~km} / \mathrm{h}$ ) and 3 days/week of aerobic activities (jogging under heart rate control - 120-140 bpm for $45 \mathrm{~min}$ ).

Results: The results showed that exercise induced a significant increase in GSR and plasma Orexin A but no significant increase in d-ROM values. Significant decreases in the serum ALT enzyme, triglycerides, and total cholesterol were found, while the HDL levels were significantly higher. Finally, a significant reduction of BMI and resting HR were reported.

Conclusion: The results of this study confirm that physical activity is associated with sympathetic activation, having a pivotal role against adverse effects linked to MetS. Moreover, this study demonstrates that, in patients with MetS, Orexin A is involved in hormonal adaptations to exercise.

Keywords: metabolic syndrome, MetS, Orexin A, body mass index, BMI, heart rate, HR, cholesterol, physical activity

\section{Introduction}

Metabolic syndrome (MetS) is a well-defined cluster of various metabolic abnormalities that come together in a single individual. Following the criteria of the International Diabetes Federation (2005), the clinical diagnosis of MetS is made when the increase of waist circumference (WC) is combined with any 2 of the following factors: lipid alteration (triglyceride levels $\geq 150 \mathrm{mg} / \mathrm{dL}$; high-density lipoprotein cholesterol $<40 \mathrm{mg} / \mathrm{dL}$ in men or $<50 \mathrm{mg} / \mathrm{dL}$ in women), blood pressure modification $(\geq 130 \mathrm{~mm} \mathrm{Hg}$ systolic or $\geq 85 \mathrm{~mm} \mathrm{Hg}$ diastolic or on hypertension treatment) or high glucose level $(\geq 100 \mathrm{mg} / \mathrm{dL}$, including diabetes) (International
Correspondence: Francesco Sessa Department of Clinical and Experimental Medicine,University of Foggia, Foggia

7II 22, Italy

Tel +39088I 736926

Email francesco.sessa@unifg.it 
Diabetes Federation). Affected individuals are most often overweight or obese. ${ }^{1-3}$ One of the most severe complications related to MetS is non-alcoholic fatty liver disease (NAFLD). ${ }^{4}$

Physical activity is an effective, practical and inexpensive way to improve health, to balance the internal homeostatic process, to control body weight and to reduce age-related cognitive decline. ${ }^{5-8}$ Particularly, aerobic exercise can greatly assist in reducing collateral effects of MetS, mainly by improving weight loss, reducing body fat, and increasing lean body mass. ${ }^{9-12}$ The data from randomized controlled trials have highlighted favorable changes in MetS criteria after aerobic training. ${ }^{13}$

During the natural physiological process of all living beings, free radicals are generated. Oxidative stress is linked to the onset of several diseases, playing a central role in the promotion of chronic and degenerative disorders as well as aging. ${ }^{14,15}$ The terms "Reactive Oxygen Species (ROS)" and "Reactive Nitrogen Species (RNS)" have been frequently used to describe free radicals and other non-radical reactive derivatives. High levels of ROS can be lethal to cells, generating modifications in cellular lipids, DNA and proteins. ${ }^{16}$ Regular physical activity helps to ease stress by decreasing pro-inflammatory mediators, even if a moderate increase in plasma ROS is considered healthy and fundamental in cellular adaptation to training. One of the unanswered questions is related to the paradox the extended muscle disuse can increase ROS production in skeletal muscle fibers as well as strenuous physical activity (contractile activity). In skeletal muscle fibers, ROS is a signaling molecule that can promote both anabolic responses (during particular conditions such as exercise training) and in other states of catabolic signaling. ${ }^{17-19}$

Moreover, the adaptive responses to sustain muscle engagement and sympathetic activation is associated with aerobic exercise. Furthermore, physical activity is related to changes in the release of Orexin A (hypocretin-1), a pleiotropic neuropeptide synthesized by neurons located in the perifornical region of the lateral hypothalamus area (LHA). Orexin A plays several roles in the regulation of various body systems. It has been found to be involved in several physiological processes, such as the regulation of food intake, ${ }^{20,21}$ arousal, metabolism, and cardiovascular and respiratory function. ${ }^{22-25}$ Moreover, it has been linked to an increase in blood pressure (BP), heart rate (HR) and metabolic rate. ${ }^{26,27}$

This study aimed to analyze the effects of physical activity, which could be considered a "natural drug", against different health problems concerning metabolism. This study aimed to analyze the effects of aerobic physical activity, which could be considered a "natural drug", against different health problems concerning metabolism. Particularly, this experimental model aimed to evaluate the beneficial effects of aerobic exercise in a cohort of MetS subjects, focusing on the role of sympathetic and orexinergic activity; the study was carried out maintaining the same diet regimen for all the study period. For this purpose, the anthropometric and biochemical parameters were analyzed to define the MetS group; moreover, to analyze the sympathetic and orexinergic system galvanic skin response, Orexin-A serum levels and heart rate measurements were tested in the same group. Indeed, as previously described, obesity, MetS, and also physical activity represent different stress conditions to active mechanisms of adaptation or re-adaptation, restoring altered homeostasis. ${ }^{28-30}$

As previously described, these parameters can be tested to evaluate the homeostasis alteration, allowing continuous measurements over a long period of time with minimal disturbance to the subject under examination. Moreover, skin conductance and heart rate variability could be used to measure the activity of the sympathetic nervous system. ${ }^{31}$ Stressful events or emergency situations may cause dynamic changes in the autonomic nervous system, in particular the activity of the sympathetic nervous system increases.

\section{Materials and Methods Subjects}

Ten male subjects (aged $54 \pm 4.16$ ) with MetS (MetS group) and ten healthy males (aged $49.7 \pm 2.79$, Healthy group) were told about the study protocol and possible risks, signed the informed consent, and voluntarily participated in the study. They were free to withdraw their participation at any time during the course of the study. The participants were enrolled in both groups if they were older than 40 years of age and had no familiarity for several conditions such as diabetes, cancer, heart disease or pathologies such as anemia, renal or liver disorders. Moreover, smokers, subjects who habitually consumed alcohol, and pregnant or lactating women were excluded. Following the scientific criteria of metabolic syndrome, ${ }^{1,2,32}$ at the start of the experimental study, all participants included in the MetS group had the coexistence of at least three factors characterizing metabolic syndrome, in particular, they were obese $\left(\mathrm{BMI}>30 \mathrm{Kg} / \mathrm{m}^{2}\right)$ with waist circumference $>102 \mathrm{~cm}$ (112.5 \pm 1.2 ), triglyceride levels $>150 \mathrm{mg} / \mathrm{dl}$, blood pressure 
$>130 / 85 \mathrm{mmHg},{ }^{33}$ and/or fasting glucose $>100 \mathrm{mg} / \mathrm{dL}$. Participants of both groups were all Caucasians, and were recruited in the same urban area and with similar social and economic status.

All procedures were performed in accordance with the Declaration of Helsinki and were approved by the Scientific Committee of the University of Foggia (Italy-FG_MetS_28/ 11/2018). Blood samples were collected from the antecubital vein into blood collection tubes containing sodium heparin for the measurement of reactive oxygen metabolites. The blood samples were centrifuged at $4^{\circ} \mathrm{C}$ immediately after collection, and the plasma was separated and stored at $-80^{\circ}$ $\mathrm{C}$ until analysis could be performed.

\section{Experimental Model}

Several blood parameters were evaluated in the MetS group and Healthy group, and are summarized in Table 1. Moreover, the same parameters were evaluated in the MetS group after 6 months of training (MetS6M group). The training protocol consists of more than $30 \mathrm{~min} /$ day of walking (each participant was free to choose their own walking speed) and 3 days/week of aerobic activities (jogging under hart rate control - 120-140 bpm for $45 \mathrm{~min}$ ), following the criteria of Physical Activity Guidelines. ${ }^{34}$ The training protocol was monitored evaluating the heart rate values with the Activio Sport System (Activio AB, Stockholm, Sweden). Moreover, heart rate, galvanic skin response, d-ROMs test, and Orexin A, were measured in the MetS6M group before and after 45 min of training in the last week of the training program.

\section{Blood Analysis}

Blood was sampled after $12 \mathrm{~h}$ of fasting.

As previously reported, the following biochemical parameters were measured on a Hitachi 911 analyzer (Roche Diagnostics, Indianapolis, USA) using Roche reagents: aspartate aminotransaminase (AST), alanine aminotransferase (ALT), gamma-glutamyl transpeptidase (GGT), cholesterol (total, HDL and LDL) and triglycerides (non-fasting). ${ }^{35}$

Serology tests (including analyses for hepatitis B and $\mathrm{C}$ viruses, and HIV virus) were negative for all patients.

\section{Blood Pressure Measurements}

Systolic blood pressure (SBP) and diastolic blood pressure (DBP) were measured to the nearest one-millimeter of mercury while the participant was seated using a sphygmomanometer and stethoscope.

\section{Resting Heart Rate (HR) Measurement}

The HR measurement was carried out with a chest strap wired to a digital R-R recorder (BTL08 SD ECG); the QRS-signal wave-form R-R signal was sampled at the resolution of $1 \mathrm{~ms}$. The HR (beats min-1) was calculated using the formula: $\mathrm{HR}=60 \mathrm{R}-\mathrm{R}$ interval-1; the $\mathrm{R}-\mathrm{R}$ interval was converted into seconds.

\section{Galvanic Skin Response (GRS)}

The GSR parameters were measured simultaneously using the SenseWear Pro Armband ${ }^{\mathrm{TM}}$ (version 3.0, BodyMedia, Inc. PA, USA), which was worn on the right arm over the

Table I Tested Parameters in Both Groups

\begin{tabular}{|c|c|c|c|c|c|}
\hline Parameters & $\begin{array}{l}\text { MetS Group (Mean } \\
\text { Values) }\end{array}$ & $\begin{array}{l}\text { MetS6M Group (Mean } \\
\text { Values) }\end{array}$ & $\begin{array}{l}\text { Healthy } \\
\text { Group }\end{array}$ & $\begin{array}{l}\text { p values MetS } \\
\text { vs HG }\end{array}$ & $\begin{array}{l}\text { p values MetS vs } \\
\text { MetS6M }\end{array}$ \\
\hline Triglycerides (mg/dl) & $199.4 \pm 16.8$ & $184.4 \pm 12.94$ & $|5| .5 \pm 8.5 \mid$ & $<0.000 I^{*}$ & $0.038 *$ \\
\hline Fasting Glucose (mg/dL) & $119.7 \pm 4.98$ & $\mid 14.7 \pm 5.31$ & $83 \pm 6.3$ & $<0.0001 *$ & $0.043 *$ \\
\hline ALT (U/L) & $50.5 \pm 11.08$ & $41.5 \pm 5.89$ & $23.1 \pm 8.65$ & $<0.000 I^{*}$ & $0.035 *$ \\
\hline AST (U/L) & $40.3 \pm 11.9$ & $37.8 \pm 10.28$ & $27.8 \pm 7.37$ & $0,011 *$ & 0.62 \\
\hline GGT (U/L) & $41.9 \pm 11.69$ & $38.8 \pm 10.09$ & $22.4 \pm 8.23$ & $0.0004^{*}$ & 0.53 \\
\hline CHOLESTEROL $(\mathrm{mg} / \mathrm{dL})$ & $215.1 \pm 14.6$ & $203.2 \pm 9.67$ & $156.9 \pm 8.9$ & $<0.000 I^{*}$ & $0.045 *$ \\
\hline $\mathrm{HDL}(\mathrm{mg} / \mathrm{dL})$ & $44.1 \pm 4.55$ & $53 \pm 6.18$ & $46.8 \pm 6.95$ & $0.04 *$ & $0.001 *$ \\
\hline $\mathrm{LDL}(\mathrm{mg} / \mathrm{dL})$ & $143.2 \pm 17.63$ & $136.8 \pm 16.1$ & $93.1 \pm 14.09$ & $<0.000 I^{*}$ & 0.4 \\
\hline BMI & $31.7 \pm 1.33$ & $29.94 \pm 1.58$ & $24.8 \pm 1.81$ & $<0.000 I^{*}$ & $0.015 *$ \\
\hline$W C(\mathrm{~cm})$ & $112.5 \pm 1.2$ & $105 \pm 1.2$ & $90 \pm 1.2$ & $<0.000 I^{*}$ & 0.05 \\
\hline Rest HR (bpm) & $82.4 \pm 3.13$ & $73.7 \pm 2.9$ & $71.1 \pm 2.18$ & $<0.0001 *$ & $<0.0001 *$ \\
\hline $\mathrm{SBP}(\mathrm{mm} \mathrm{Hg})$ & $139.7 \pm 4.9$ & $136.7 \pm 4.69$ & $|24.3 \pm 5.3|$ & $<0.000 I^{*}$ & 0.18 \\
\hline $\mathrm{DBP}(\mathrm{mm} \mathrm{Hg})$ & $90 \pm 4.1$ & $86.6 \pm 4.35$ & $77.2 \pm 3.4$ & $<0.0001 *$ & 0.08 \\
\hline
\end{tabular}

Note: *The last columns report the results of the p-value of the $t$-test. A p-value less $\leq 0.05$ (in bold) was considered statistically significant.

Abbreviations: ALT, alanine aminotransferase; AST, aspartate aminotransferase; GGT, gamma glutamyl transferase; HDL, high-density lipoprotein; LDL, low-density lipoprotein; BMI, body mass index; WC, Waist circumference; Rest HR, rest heart rate; SBP, systolic blood pressure; DBP, diastolic blood pressure. 
triceps muscle at the midpoint between the acromion and olecranon processes, as recommended by the manufacturer. The SenseWear Pro Armband is a portable sensing device, $4.0 \mathrm{~cm}$ diameter and $1.9 \mathrm{~cm}$ high and $34 \mathrm{~g}$ in weight that has 4 sensors: - Accelerometer (3 axes); • Heat flow; - Skin temperature; - GSR. All physiological data were then processed by advanced algorithms to calculate and report total energy expenditure, metabolic physical activity, and sleep. However, in this study, the data about GSR parameters were collected at basal level (time 0 , at rest before physical activity), after $45 \mathrm{~min}$ (at the end of training), and after $60 \mathrm{~min}$. (15 min after training).

\section{Plasma Orexin A Detection}

Blood samples were withdrawn from a forearm vein, at 8:00 a.m. after an overnight fast, into Vacutainer tubes (BD, Franklin Lakes, NJ, USA) containing EDTA and $0.45 \mathrm{TIU} / \mathrm{mL}$ of aprotinin. After centrifugation at $3000 \mathrm{rpm}$ for $12 \mathrm{~min}$ at $4^{\circ} \mathrm{C}$, the plasma was separated and stored at $-80^{\circ} \mathrm{C}$ until analysis. Enzyme-linked immunoassay kits (ELISA) were used to measure plasma Orexin A levels. Hypocretin Orexin A1 ELISA kits were purchased from Phoenix Pharmaceuticals. Before measurement, plasma Orexin A was extracted using Sep-Pak $\mathrm{C}_{18}$ columns (Waters, Milford, MA, USA), $10 \mathrm{~mL}$ of methanol and $20 \mathrm{~mL}$ of $\mathrm{H}_{2} \mathrm{O}$ were used to activate the columns. The following step consisted of adding 1-2 mL of the sample to the column and washing with $20 \mathrm{~mL}$ of water. Samples were eluted slowly with $80 \%$ acetonitrile and the resulting volume was reduced to $400 \mu \mathrm{L}$ under nitrogen flow. An aliquot was used for exsiccation using the Speedvac (Savant Instruments, Holbrook, NY, USA). The dry residue was dissolved in water and used for the ELISA test. No cross-reactivity for hypocretin-1, hypocretin-2, and agouti-related protein amide had been previously reported. The kit used for the quantification has an intra-assay error $<5 \%$ and an inter-assay error $<14 \%$. The detection concentration limit was $0.37 \mathrm{ng} / \mathrm{mL}$.

\section{The d-ROMs Test}

The d-ROMs test was performed using the FRAS4 system according to the manufacturer's instructions (Wismerll Co., Tokyo, Japan). For each participant, $20 \mu \mathrm{L}$ plasma was tested, and tests were run in duplicate. The results obtained from the d-ROMs test were expressed in arbitrary units called "Carratelli units" (U.CARR). The results were presented and analyzed as mean values.

\section{Statistical Analysis}

Data are presented as mean \pm standard deviation (SD). Statistical analysis of all parameters is summarized in Table 1 and was performed using GraphPad Prism 5 software (GraphPad software, Inc, La Jolla, CA, USA) and by a researcher trained in biostatistics. Two-tailed Student's $t$-test was used. A p-value of $<0.05$ was considered statistically significant. GSR, plasma Orexin A and d-ROMs values were subjected to a one-way analysis of variance ANOVA with Tukey HSD post hoc comparisons. The analysis was performed using SPSS software version 24 (IBM Japan, Tokyo, Japan), and the statistical level of significance was set at 0.05 .

\section{Results}

The mean values of the blood parameters tested are summarized in Table 1, as well as the mean values of resting HR, BMI, and blood pressure.

The statistical analysis performed with Student's $t$-test demonstrated significant differences between MetS and Healthy groups for all parameters: all values were statistically different. The results of Student's $t$-test comparing the data of the MetS and Mets6M groups are interesting; after 6 months of training, all parameters were improved in the MetS6M group, even if 7 parameters had statistical differences. The blood values of triglycerides, fasting glucose, ALT, total cholesterol and HDL levels were statistically improved; moreover, the parameters of BMI and resting HR were significantly decreased.

As shown in Figure 1, resting HR improved after 6 months of aerobic training; the positive effects of physical activity are shown in the BMI values: the mean values of the MetS group are located in the obesity category (Class 1 obesity), while after a period of aerobic training the MetS group improved this parameter including the participants in the overweight category (Figure 2).

In the last week of the training program, GSR, plasma Orexin A and d-ROMs were tested in the MetS6M group.

Figure 3 shows GSR changes. Physical activity caused an increase in GSR with the maximum level at $45 \mathrm{~min}$. GSR $(\mu \mathrm{S})$ increased from a basal value of $1.28 \pm 0.09$ to $1.69 \pm 0.07$ at $45 \mathrm{~min}$, and it decreased to $1.6 \pm 0.04$ at 60 min. The ANOVA test showed significant effects $[F(2,27)$ $\left.=3.35, \mathrm{p}<0.05,1.8 \times 10^{-12}\right]$. The post hoc test showed a difference between pre-exercise and post-exercise values at $0 \mathrm{~min} ., 45 \mathrm{~min}$., and $60 \mathrm{~min}$. 


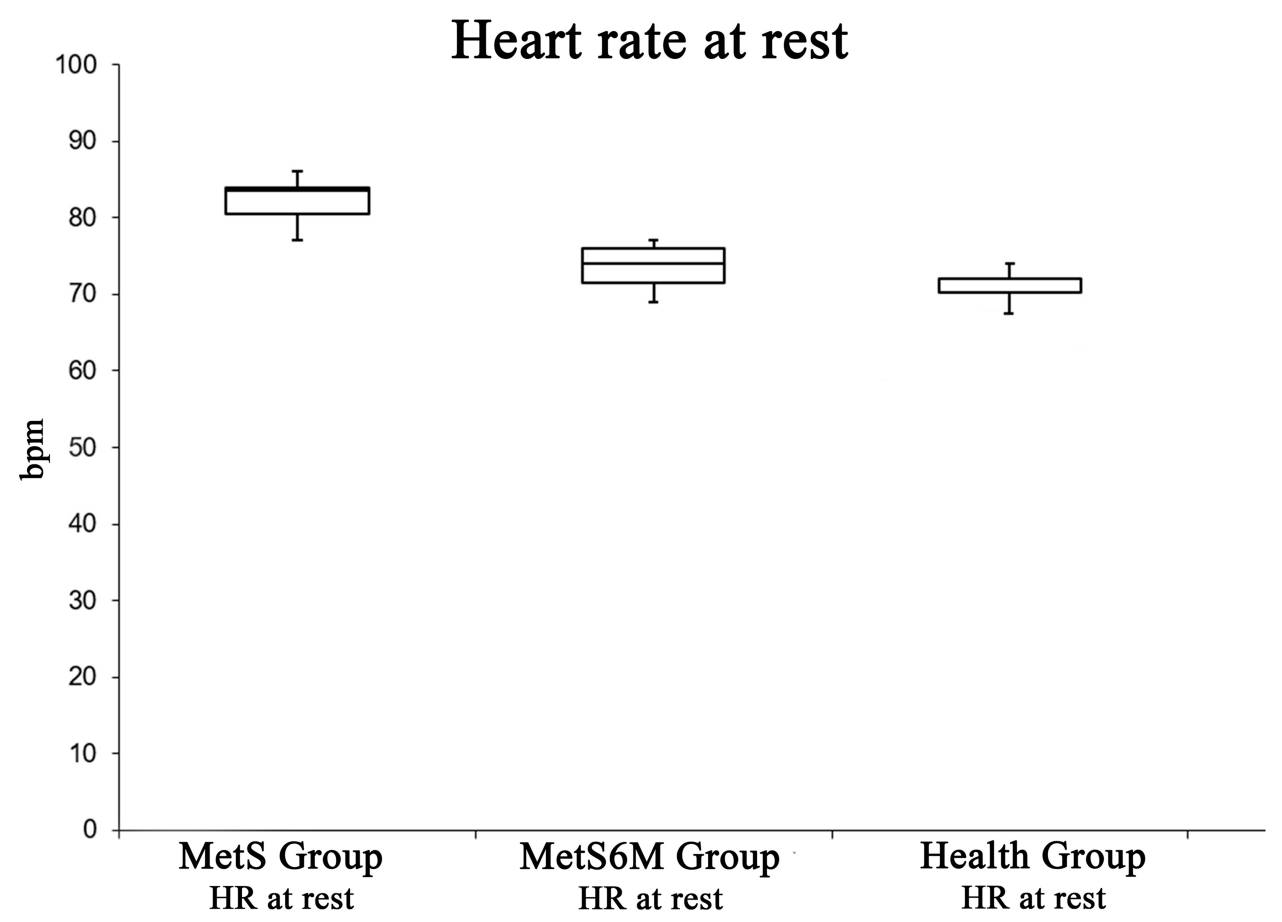

Figure I Resting HR is statistically higher in the MetS Group with respect to the Healthy Group. Moreover, this parameter is significantly improved after 6 months aerobic training.

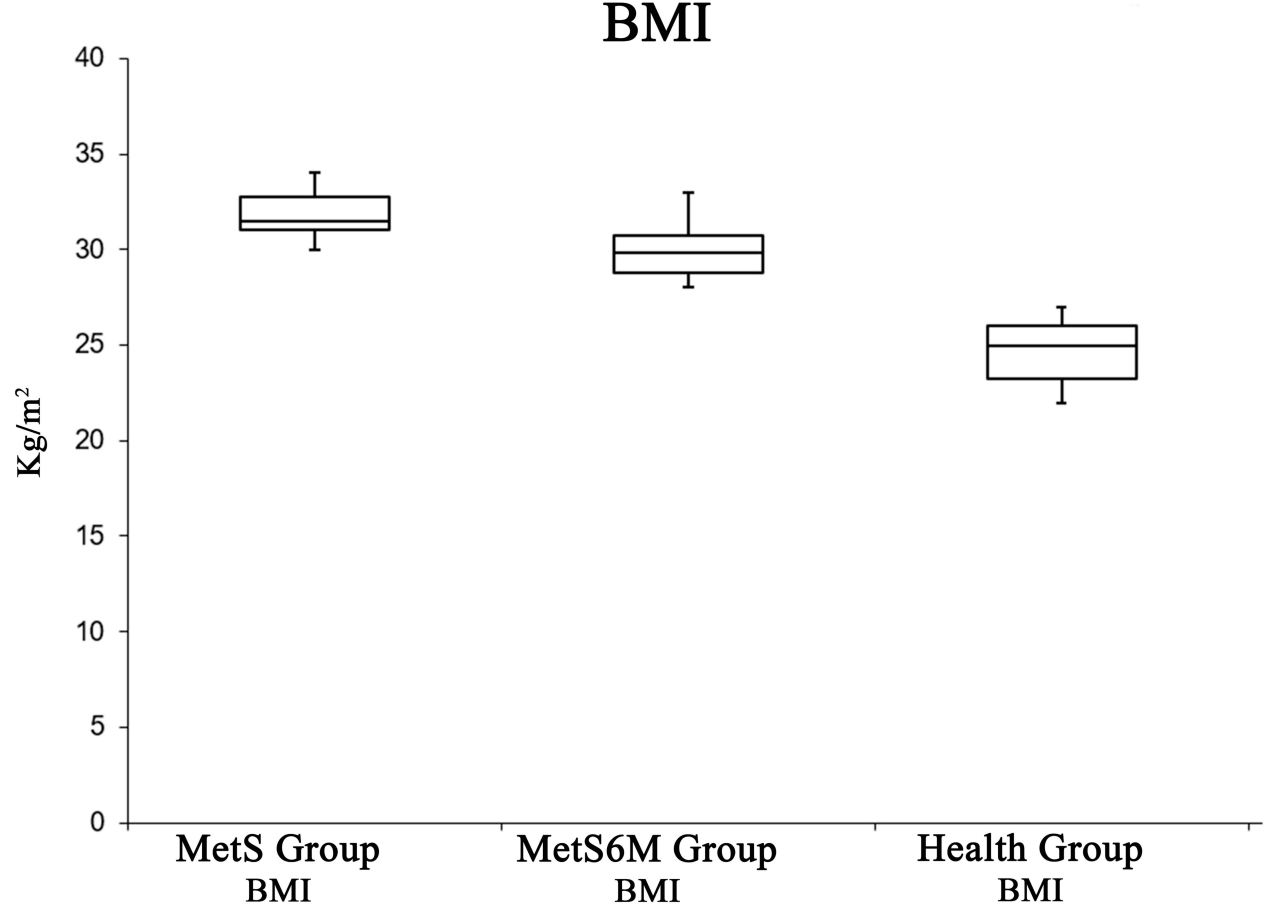

Figure $2 \mathrm{BMI}$ is statistically higher in the MetS Group related to the Healthy Group. Moreover, this parameter is significantly improved after 6 months aerobic training.

Figure 4 shows Orexin A changes. Physical activity caused an increase in Orexin A, from a basal level of 2700 $\pm 72.26 \mathrm{pg} / \mathrm{mL}$ to a maximum level $(3626 \pm 95.93 \mathrm{pg} / \mathrm{mL})$ at $45 \mathrm{~min}$. Orexin A decreased to a value of $3196 \pm 60.22$ at $60 \mathrm{~min}$. The ANOVA test showed significant differences $\left[F(2,27)=3.35, \mathrm{p}<0.05,3.83 \times 10^{-20}\right]$. The post hoc test 


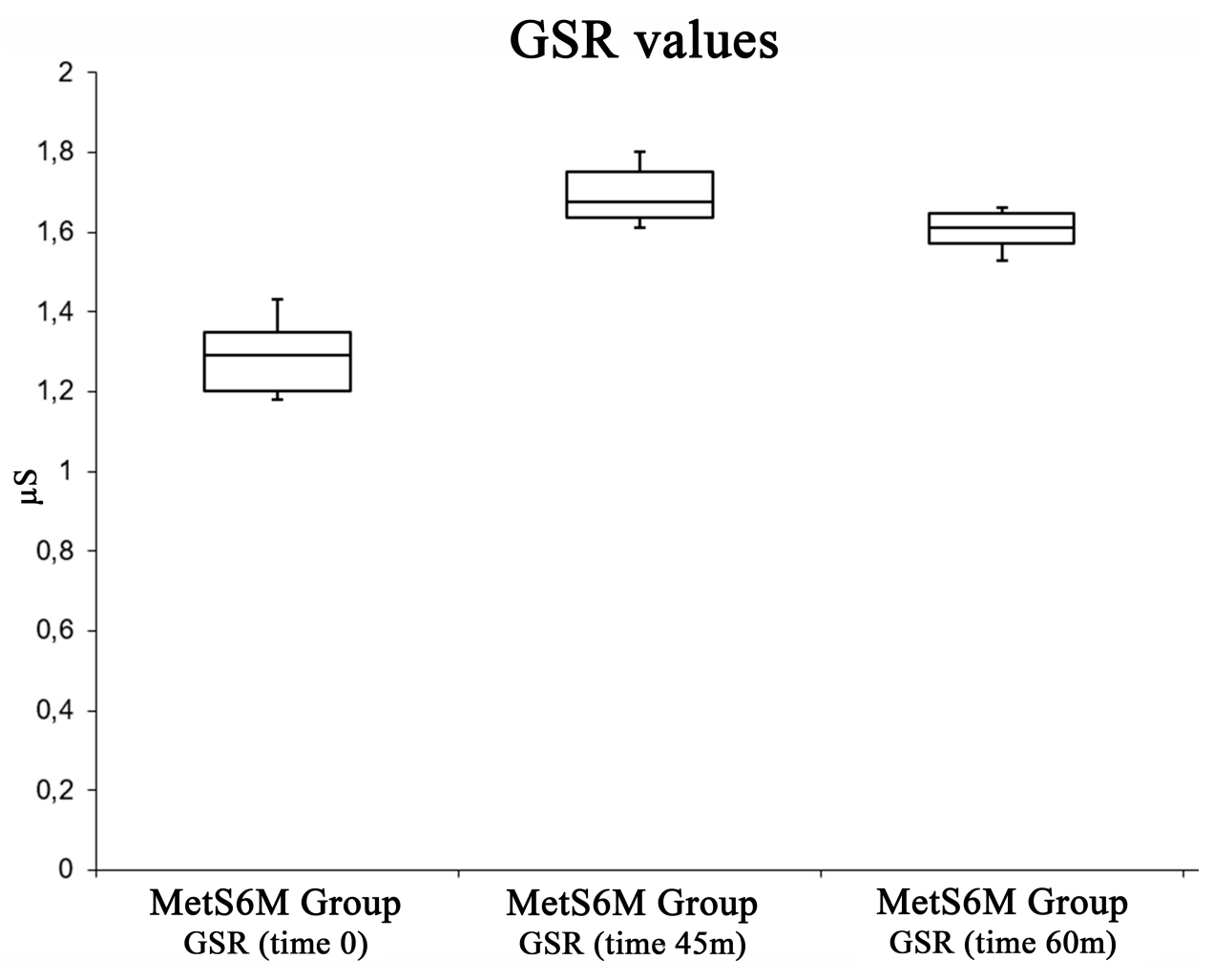

Figure 3 Box plot analysis summarizing GSR variations among pre-exercise status, at the end of the exercise status.

\section{Orexin A values}

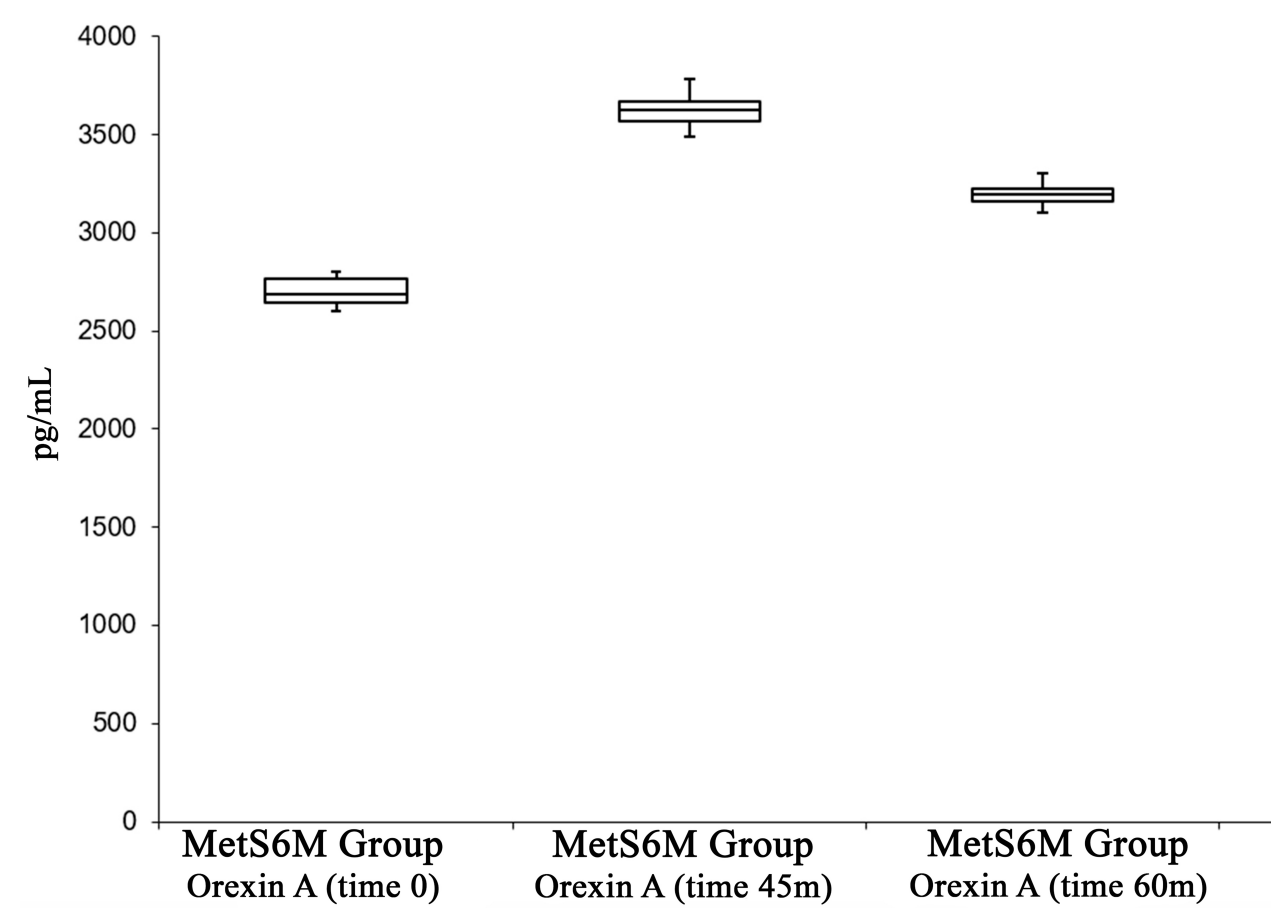

Figure 4 Box plot analysis summarizing Orexin A variations among pre-exercise status, at the end of the exercise status and in the recovery periods. 
showed a difference between pre-exercise and postexercise values at $0 \mathrm{~min}$., $45 \mathrm{~min}$., and $60 \mathrm{~min}$.

Figure 5 shows d-ROMs changes. Physical activity did not cause an increase in d-ROMs from a basal value of $284 \pm 12.97 \mu$ Carr to a maximum level $(292.4 \pm 6.51$ $\mu$ Carr) at 45 min. d-ROMs decreased to a value of 285.7 \pm 13.03 at $60 \mathrm{~min}$. The ANOVA test did not show significant differences $[\mathrm{F}(2,27)=3.35, \mathrm{p}=0.24]$.

\section{Discussion and Conclusion}

Characteristics of subjects with and without MetS have been extensively described: MetS is defined as the contemporary presence of several interconnected human diseases; physiological, biochemical, clinical, and metabolic factors can be combined, increasing the risk of atherosclerotic cardiovascular disease and diabetes; in particular cases, all these factors can cause the "exitus" of the subject. $^{36-39}$ As shown in Table 1, the participants included in our study group had several parameters that confirmed the diagnoses of MetS: compared to the healthy group the values were significantly higher.

The guidelines on physical activity recommend a daily minimum of $30 \mathrm{~min}$ of moderate-intensity physical activity, in order to prevent organ damage and cardiovascular diseases. However, several experimental studies have demonstrated that 60 min of moderate-intense brisk walking could be very useful to improve several clinical parameters, especially in people living in developed countries. $^{40-43}$ In our experimental model, we tested several blood parameters after 6 months of regular physical activity without dietary changes. It was very interesting that all blood parameters tested were improved with respect to the levels of the same parameters tested at time 0 . The results of the present study show the positive effects of physical activities as "natural drugs" against metabolic syndrome. The results of this study confirm that physical activity is associated with sympathetic activation, as demonstrated by HR and GSR increases during exercise. The association between Orexin A plasma levels and sympathetic discharge is also described in this study. Physical activity induces an increase in plasmatic Orexin $\mathrm{A},{ }^{22}$ as underlined by the results of the present experiment. Moreover, an adaptation of HR to physical activities is described in the results section. The present study suggests a correlation between plasmatic Orexin A and HR adaptation, as an expression of sympathetic activity.

As previously described, Orexin A affects both peripheral energy balance and the central nervous system, particularly

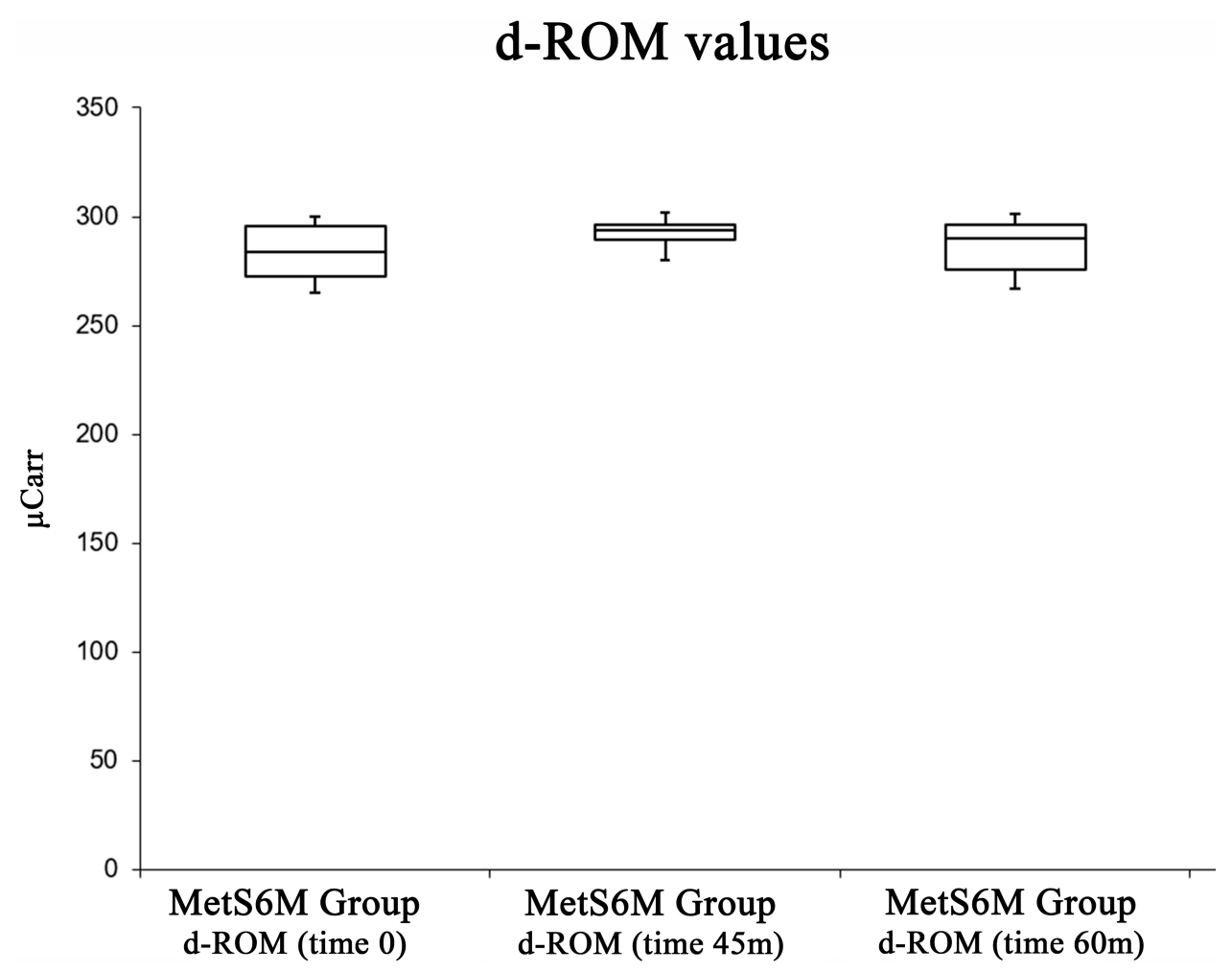

Figure 5 Box plot analysis summarizing d-ROMs variations among pre-exercise status, at the end of the exercise status and in the recovery periods. 
when the subject is under stress; moreover, motivated behaviors (for example, food intake) and sleep-wakefulness can be coordinated by this neuropeptide. ${ }^{7,44}$ Orexin 1 receptor (OX1R) has been reported in various tissues such as the reproductive system, intestine, adrenal glands, pancreas, and kidney: binding its receptor, Orexin A has several pleiotropic functions. ${ }^{15}$ Several vital body functions such as sleep/ wake states, eating behavior, reward systems, energy homeostasis, cognition, and mood could be influenced by Orexin A levels. Likewise, it plays an important role in energy balance and obesity. ${ }^{45-47}$ Considering the important functions of the orexinergic system, it is well known that dysfunction in this system may be related to various pathological conditions. ${ }^{7,48}$ Moreover, to the best of our knowledge, this study is the first to demonstrate that, in patients with MetS, Orexin A is involved in hormonal adaptations to exercise, indicating that this peptide controls several adaptations (such as cardiovascular changes) to physical activity also in MetS.

In addition, significant decreases in the serum ALT enzyme, triglycerides, fasting glucose, and total cholesterol were found, while the HDL levels were significantly higher. It is well described that aerobic activity induces positive effects on HDL plasma levels, depending on the entry level. ${ }^{49}$ Moreover, the low plasma levels of HDL were associated with high levels of triglycerides. In agreement with Couillard et al, the results of the present study confirmed that aerobic activity may be related to the reduction of triglyceride levels combined with improved HDL levels. ${ }^{50}$

Physical activity is not considered the only way to fight MetS: the best approach to produce lifestyle modification treatment involves a multidisciplinary team composed of physicians and non-physician health professionals. To date, professionals with a master degree in exercise physiology should be collaborating with other specialists such as biologists, dieticians, and behavioral psychologists. Moreover, lifestyle modification is a complex route that should lead subjects to modify their habits both related to diet and physical activities. In this way, an ambitious goal could be achieved, reducing all risk factors linked with metabolic syndrome. ${ }^{43,51-53}$

Finally, the results of the present study show that physical activity did not cause an increase in d-ROMs values in the study group. Previous studies have demonstrated that physical exercise increases the metabolic demands of skeletal muscles leading to greater oxygen uptake and blood flow to the muscles and other organs ${ }^{54,55}$ thus leading to a higher ROS generation. ${ }^{35,47,48}$ According to the "mitochondrial hypothesis of aging", mitochondria are the main source of
ROS generation, especially during exercise, and their generating rate is related to mitochondrial oxygen consumption. ${ }^{56}$ Nevertheless, the presented results highlight that these modifications described in athlete groups are not presented in our study group, suggesting that the status of MetS negatively influenced the beneficial effects of physical activity. In agreement with our results, several studies demonstrate that exercise leads to oxidative stress only when it is exhaustive due to an increase in lipid peroxidation products and, consequently, oxidative stress and damage of muscle mitochondrial membranes. ${ }^{57-62}$

The main limitation of the present study is linked to the small number (10 for both groups) of participants. Moreover, the choice of comparing the positive effects of physical activity in the same group at different times (MetS vs MetS6M), on the one hand, represents an advantage allowing comparison in a homogeneous group; on the other hand, it undoubtedly removed the MetS group from the final evaluation. Furthermore, the exercise effects on the control group were not evaluated. Finally, the results concerning oxidative stress and sympathetic activity should be confirmed increasing the number of tested markers. Moreover, an added limitation of the present study is that there is not a pre-post 6 months' exercise comparison in the healthy group.

For these reasons, further experiments will have to be performed to verify these hypotheses; moreover, it could be interesting to explore the role of Orexin A on the peripheral adaptations to physical activity and oxidative stress levels: these studies could confirm the multitasking role of this neuropeptide. Indeed, physical activity is associated with sympathetic activation, having a pivotal role against adverse effects linked to MetS. Moreover, this study demonstrates that, in patients with MetS, Orexin A is involved in hormonal adaptations to exercise. In addition, the exercise induced a significant increase in GSR, and plasma Orexin A, but no significant increase of d-ROM values, and an amelioration in anthropometric and biochemical parameters such as glycemic and lipid profile. For these reasons, physical activity, in particular aerobic exercise, may be considered a valid strategy to improve health status without changes in diet in metabolic syndrome.

\section{Disclosure}

The authors report no conflicts of interest in this work. 


\section{References}

1. Grundy SM, Brewer HB, Cleeman JI, Smith SC, Lenfant C. Definition of metabolic syndrome: report of the National Heart, Lung, and Blood Institute/American Heart Association conference on scientific issues related to definition. Circulation. 2004;109 (3):433-438.

2. Kaur J. A comprehensive review on metabolic syndrome. Cardiol Res Pract. 2014;2014:1-21. doi:10.1155/2014/943162

3. Huang PL. A comprehensive definition for metabolic syndrome. Dis Model Mech. 2009;2(5-6):231-237. doi:10.1242/dmm.001180

4. Byrne CD, Patel J, Scorletti E, Targher G. Tests for diagnosing and monitoring non-alcoholic fatty liver disease in adults. $B M J$. 2018;362. Available from: https://www.scopus.com/inward/record uri ? eid $=2$-s $2.0-85050204997 \&$ doi $=10.1136 \% 2 \mathrm{Fbmj}$. k2734\&partnerID $=40 \& m d 5=a e b 23 f d a 673 a 2 f b 5 f f 83173 b 00797 b 39$.

Accessed June 24, 2020.

5. Obici S, Magrisso IJ, Ghazarian AS, et al. Moderate voluntary exercise attenuates the metabolic syndrome in melanocortin-4 receptor-deficient rats showing central dopaminergic dysregulation. Mol Metab. 2015;4(10):692-705. doi:10.1016/j.molmet.2015.07.003

6. Sessa F, Messina G, Valenzano A, et al. Sports training and adaptive changes. Sport Sci Health. 2018;14(3):705-708. doi:10.1007/s11332018-0464-z

7. Chieffi S, Messina G, Villano I, et al. Neuroprotective effects of physical activity: evidence from human and animal studies. Front Neurol. 2017;8. doi:10.3389/fneur.2017.00188

8. Wiklund P. The role of physical activity and exercise in obesity and weight management: time for critical appraisal. J Sport Health Sci. 2016;5(2):151-154. doi:10.1016/j.jshs.2016.04.001

9. Normandin E, Chmelo E, Lyles MF, Marsh AP, Nicklas BJ. Effect of resistance training and caloric restriction on the metabolic syndrome. Med Sci Sports Exerc. 2017;49(3):413-419. doi:10.1249/MSS.0000 000000001122

10. Donini LM, Cuzzolaro M, Gnessi L, et al. Obesity treatment: results after 4 years of a nutritional and psycho-physical rehabilitation program in an outpatient setting. Eat Weight Disord. 2014;19 (2):249-260. doi:10.1007/s40519-014-0107-6

11. Van Der Windt DJ, Sud V, Zhang H, Tsung A, Huang H. The effects of physical exercise on fatty liver disease. Gene Expr. 2018;18 (2):89-101. doi:10.3727/105221617X15124844266408

12. Sessa F, Anna V, Messina G, et al. Heart rate variability as predictive factor for sudden cardiac death. Aging (Albany NY). 2018;10 (2):166-177. doi:10.18632/aging.101386

13. Messina G, Vicidomini C, Viggiano A, et al. Enhanced parasympathetic activity of sportive women is paradoxically associated to enhanced resting energy expenditure. Auton Neurosci Basic Clin. 2012;169(2):102-106. doi:10.1016/j.autneu.2012.05.003

14. Dröge W. Free radicals in the physiological control of cell function. Physiol Rev. 2002;82(1):47-95. doi:10.1152/physrev.00018.2001

15. Phull A-R, Nasir B, Ul Haq I, Kim SJ. Oxidative stress, consequences and ROS mediated cellular signaling in rheumatoid arthritis. Chem Biol Interact. 2018;281:121-136. doi:10.1016/j.cbi.20 17.12.024

16. Simioni C, Zauli G, Martelli AM, et al. Oxidative stress: role of physical exercise and antioxidant nutraceuticals in adulthood and aging. Oncotarget. 2018;9(24):17181-17198. doi:10.18632/oncotarget.24729

17. Davalli P, Mitic T, Caporali A, Lauriola A, D'Arca D. ROS, cell senescence, and novel molecular mechanisms in aging and age-related diseases. Oxid Med Cell Longev. 2016;2016:1-18. doi: 10.1155/2016/3565127

18. Messina A, Monda M, Valenzano A, et al. Functional changes induced by orexin a and adiponectin on the sympathetic/parasympathetic balance. Front Physiol. 2018;9. doi:10.3389/fphys.20 18.00259
19. Powers SK, Duarte J, Kavazis AN, Talbert EE. Reactive oxygen species are signalling molecules for skeletal muscle adaptation. Exp Physiol. 2010;95(1):1-9. doi:10.1113/expphysiol.2009.050526

20. Monda V, Salerno M, Fiorenzo M, et al. Role of sex hormones in the control of vegetative and metabolic functions of middle-aged women. Front Physiol. 2017;8(OCT). doi:10.3389/fphys.2017.00773

21. Messina A, Monda V, Avola R, et al. Role of the orexin system on arousal, attention, feeding behaviour and sleep disorders. Acta Medica Mediterr. 2017;33(4):645-649.

22. Messina G, Di Bernardo G, Viggiano A, et al. Exercise increases the level of plasma orexin A in humans. J Basic Clin Physiol Pharmacol. 2016;27(6):611-616. doi:10.1515/jbcpp-2015-0133

23. Marra ML, Valenzano A, Ruberto M, et al. The effects of overweight and obesity on cognitive functions and psychological well-being. Acta Medica Mediterr. 2017;33:1225-1231.

24. Monda V, Villano I, Messina A, et al. Exercise modifies the gut microbiota with positive health effects. Oxid Med Cell Longev. 2017;2017:1-8. doi:10.1155/2017/3831972

25. Monda V, Salerno M, Sessa F, et al. Functional changes of orexinergic reaction to psychoactive substances. Mol Neurobiol. 2018;55(8):1-7.

26. Shirasaka T, Nakazato M, Matsukura S, Takasaki M, Kannan H. Sympathetic and cardiovascular actions of orexins in conscious rats. Am J Physiol Regul Integr Comp Physiol. 1999;277(6):R1780 R1785. doi:10.1152/ajpregu.1999.277.6.R1780

27. Hart EC, Head GA, Carter JR, et al. Recording sympathetic nerve activity in conscious humans and other mammals: guidelines and the road to standardization. Am J Physiol Heart Circ Physiol. 2017;312 (5):H1031-H1051. doi:10.1152/ajpheart.00703.2016

28. Holvoet P. Stress in obesity and associated metabolic and cardiovascular disorders. Scientifica (Cairo). 2012;2012:1-19. doi:10.6064/ 2012/205027

29. Chaput J-P, Doucet É, Tremblay A. Obesity: a disease or a biological adaptation? An update. Obes Rev. 2012;13(8):681-691. doi:10.1111/ j.1467-789X.2012.00992.x

30. Silverman MN, Deuster PA. Biological mechanisms underlying the role of physical fitness in health and resilience. Interface Focus. 2014;4(5):20140040. doi:10.1098/rsfs. 2014.0040

31. Mackersie CL, Calderon-Moultrie N. Autonomic nervous system reactivity during speech repetition tasks: heart rate variability and skin conductance. Ear Hear. 2016;37:118S-125S. doi:10.1097/ AUD.0000000000000305

32. Huang Y, Mahley RW. Apolipoprotein E: structure and function in lipid metabolism, neurobiology, and alzheimer's diseases. Neurobiol Dis. 2014;72:3-12. doi:10.1016/j.nbd.2014.08.025

33. Things you need to know about blood pressure and hypertension. Can J Cardiol. 2006;22(7):601-602.

34. 2018 Physical Activity Guidelines Advisory Committee. 2018 physical activity guidelines advisory committee scientific report. US Dep Heal Hum Serv. 2018.

35. Valenzano A, Polito R, Trimigno V, et al. Effects of very low calorie ketogenic diet on the orexinergic system, visceral adipose tissue, and ROS production. Antioxidants. 2019;8(12):643. doi:10.3390/antiox8120643

36. Chieffi S, Messina G, Villano I, et al. Exercise influence on hippocampal function: possible involvement of orexin-a. Front Physiol. 2017;8. doi:10.3389/fphys.2017.00085

37. Grundy SM, Cleeman JI, Daniels SR, et al. Diagnosis and management of the metabolic syndrome an American Heart Association/ National Heart, Lung, and Blood Institute scientific statement. Curr Opin Cardiol. 2006.

38. O'Neill S, O'Driscoll L. Metabolic syndrome: a closer look at the growing epidemic and its associated pathologies. Obes Rev. 2015;16 (1):1-12. doi:10.1111/obr.12229

39. Ritchie SA, Connell JMC. The link between abdominal obesity, metabolic syndrome and cardiovascular disease. Nutr Metab Cardiovasc Dis. 2007;17(4):319-326. doi:10.1016/j.numecd.2006. 07.005 
40. Thompson PD, Buchner D, Piña IL, et al. Exercise and physical activity in the prevention and treatment of atherosclerotic cardiovascular disease: a statement from the Council on Clinical Cardiology (subcommittee on exercise, rehabilitation, and prevention) and the Council on Nutrition, Physical. Circulation. 2003;107(24):31 09-3116. doi:10.1161/01.CIR.0000075572.40158.77

41. Steinberger J, Daniels SR, Eckel RH, et al. Progress and challenges in metabolic syndrome in children and adolescents. A scientific statement from the American Heart Association atherosclerosis, hypertension, and obesity in the young committee of the Council on Cardiovascular Disease in the Young. Circulation. 2009;119 (4):628-647. doi:10.1161/CIRCULATIONAHA.108.191394

42. Messina A, Monda V, Sessa F, et al. Sympathetic, metabolic adaptations, and oxidative stress in autism spectrum disorders: how far from physiology? Front Physiol. 2018;9(MAR). doi:10.3389/fphys.2018. 00261

43. Monda V, Villano I, Messina A, et al. Aerobic exercise and orexin A: role of sympathetic activity and redox system. J Biol Regul Homeost Agents. 2019;33(2):587-592.

44. Tsujino N, Sakurai T. Orexin/hypocretin: a neuropeptide at the interface of sleep, energy homeostasis, and reward system. Pharmacol Rev. 2009;61(2):162-176. doi:10.1124/pr.109.001321

45. Perez-Leighton CE, Billington CJ, Kotz CM. Orexin modulation of adipose tissue. Biochim Biophys Acta Mol Basis Dis. 2014;1842 (3):440-445. doi:10.1016/j.bbadis.2013.06.007

46. Polito R, Nigro E, Messina A, et al. Adiponectin and orexin-A as a potential immunity link between Adipose tissue and central nervous system. Front Physiol. 2018;9. doi:10.3389/fphys.2018.00982

47. Salerno M, Villano I, Nicolosi D, et al. Modafinil and orexin system: interactions and medico-legal considerations. Front Biosci. 2019; 24:564-575.

48. Sperandeo R, Maldonato MN, Messina A, et al. Orexin system: network multi-tasking. Acta Medica Mediterr. 2018;34(2):349-356.

49. Williams PT, Stefanick ML, Vranizan KM, Wood PD. The effects of weight loss by exercise or by dieting on plasma high-density lipoprotein (HDL) levels in men with low, intermediate, and normal-tohigh HDL at baseline. Metabolism. 1994;43(7):917-924. doi:10.101 6/0026-0495(94)90277-1

50. Couillard C, Després J-P, Lamarche B, et al. Effects of endurance exercise training on plasma HDL cholesterol levels depend on levels of triglycerides. Arterioscler Thromb Vasc Biol. 2007.
51. Dongiovanni P, Lanti C, Riso P, Valenti L. Nutritional therapy for nonalcoholic fatty liver disease. J Nutr Biochem. 2016;29:1-11. doi:10.1016/j.jnutbio.2015.08.024

52. Vestbo J, Hurd SS, Agustí AG, et al. Global strategy for the diagnosis, management, and prevention of chronic obstructive pulmonary disease GOLD executive summary. Am J Respir Crit Care Med. 2013;187(4):347-365. doi:10.1164/rccm.201204-0596PP

53. Monda M, Messina G, Scognamiglio I, et al. Short-term diet and moderate exercise in young overweight men modulate cardiocyte and hepatocarcinoma survival by oxidative stress. Oxid Med Cell Longev. 2014;2014.

54. Sallam N, Laher I. Exercise modulates oxidative stress and inflammation in aging and cardiovascular diseases. Oxid Med Cell Longev. 2016;2016:1-32. doi:10.1155/2016/7239639

55. Li G, He H. Hormesis, allostatic buffering capacity and physiological mechanism of physical activity: a new theoretic framework. Med Hypotheses. 2009;72(5):527-532. doi:10.1016/j.mehy.2008.12.037

56. Navarro A, Gomez C, López-Cepero JM, Boveris A. Beneficial effects of moderate exercise on mice aging: survival, behavior, oxidative stress, and mitochondrial electron transfer. Am J Physiol Integr Comp Physiol. 2004;286(3):R505-R511. doi:10.1152/ajpregu.00208.2003

57. Radák Z, Naito H, Kaneko T, et al. Exercise training decreases DNA damage and increases DNA repair and resistance against oxidative stress of proteins in aged rat skeletal muscle. Pflugers Arch. 2002;445 (2):273-278. doi:10.1007/s00424-002-0918-6

58. Apel K, Hirt H. Reactive oxygen species: metabolism, oxidative stress, and signal transduction. Annu Rev Plant Biol. 2004;55 (1):373-399. doi:10.1146/annurev.arplant.55.031903.141701

59. Jackson MJ. Control of reactive oxygen species production in contracting skeletal muscle. Antioxid Redox Signal. 2011;15(9):24 77-2486. doi:10.1089/ars.2011.3976

60. Cazzola R, Russo-Volpe S, Cervato G, Cestaro B. Biochemical assessments of oxidative stress, erythrocyte membrane fluidity and antioxidant status in professional soccer players and sedentary controls. Eur J Clin Invest. 2003;33(10):924-930. doi:10.1046/ j.1365-2362.2003.01227.x

61. Rizzo AM, Corsetto PA, Montorfano G, et al. Effects of long-term space flight on erythrocytes and oxidative stress of rodents. PLoS One. 2016;7(3):e32361. doi:10.1371/journal.pone.0032361

62. Finaud J, Lac G, Filaire E. Oxidative stress: relationship with exercise and training. Sports Med. 2006;36(4):327-358. doi:10.2165/ 00007256-200636040-00004

Diabetes, Metabolic Syndrome and Obesity: Targets and Therapy

\section{Publish your work in this journal}

Diabetes, Metabolic Syndrome and Obesity: Targets and Therapy is an international, peer-reviewed open-access journal committed to the rapid publication of the latest laboratory and clinical findings in the fields of diabetes, metabolic syndrome and obesity research. Original research, review, case reports, hypothesis formation, expert opinion and commentaries are all considered for publication. The manuscript management system is completely online and includes a very quick and fair peer-review system, which is all easy to use. Visit http://www.dovepress.com/testimonials.php to read real quotes from published authors. 